\title{
Morphological and Molecular Characterization of Globodera Populations from Oregon and Idaho
}

\author{
A. M. Skantar, Z. A. Handoo, I. A. Zasada, R. E. Ingham, L. K. Carta, and D. J. Chitwood
}

First, second, fifth, and sixth authors: United States Department of Agriculture-Agricultural Research Service (USDA-ARS) Nematology Laboratory, Beltsville, MD 20705; third author: USDA-ARS Horticultural Crops Research Laboratory, Corvallis, OR 97330; and fourth author: Department of Botany and Plant Pathology, Oregon State University, Corvallis 97331.

Accepted for publication 30 November 2010.

\begin{abstract}
Skantar, A. M., Handoo, Z. A., Zasada. I. A., Inghram, R. E., Carta, L. K., and Chitwood, D. J. 2011. Morphological and molecular characterization of Globodera populations from Oregon and Idaho. Phytopathology 101:480-491.

An unusual population of cyst nematode was found in soils collected from a Powell Butte, OR field with a cropping history including potato, wheat, other crops, and significant weed presence. These nematodes could not be placed with certainty into any known species and exhibited some unique morphological features in some specimens. Compared with Globodera pallida, the cyst body length was slightly longer and the second-stage juvenile stylet length was slightly shorter. In some individuals, the $\mathrm{J} 2$ stylet knob height was greater and the tail annules were more prominent than in G. pallida, and the tail abruptly narrowed, with a slight constriction near the posterior third of the hyaline terminus.

some individuals, the tail termini of second-stage juveniles were more bluntly pointed, and the stylet knobs were more anteriorly directed with greater height. Unlike G. tabacum, the cyst wall often lacked a networklike pattern and, in some individuals, the juvenile tail terminus distinctly narrowed after a constriction. Molecularly, the population was distinct from G. pallida, G. rostochiensis, and G. tabacum. Multiplex polymerase chain reaction of the internal transcribed spacer (ITS) rDNA region gave results similar to G. tabacum; however, ITS restriction fragment length polymorphism patterns were observed to have individual bands in common with $G$. rostochiensis and G. pallida. Phylogenetic analysis based on ITS1 and -2 rDNA sequences showed greatest similarity to populations from Argentina and Chile; together, they form a moderately supported clade, distinct from $G$. rostochiensis, G. tabacum, $G$. "mexicana," European type G. pallida, and several G. pallida populations from South America.
\end{abstract} Compared with $G$. rostochiensis, the hyaline tail terminus had a larger number of refractive bodies, and cysts of this population had a smaller Granek's ratio and fewer cuticular ridges between the anus and vulva. In
Additional keywords: detection, diagnosis, morphology, PCR, potato, RFLP, Solanum tuberosum, taxonomy.
The pale potato cyst nematode Globodera pallida (Stone 1973) Behrens 1975 and the golden potato cyst nematode $G$. rostochiensis (Wollenweber 1923) Behrens 1975 are regulated pathogens of potato, with a United States market valued at nearly $\$ 3.9$ billion (3). Collectively, these species are referred to as potato cyst nematodes (PCNs). Within the United States, G. rostochiensis was first found in New York in 1941 and has been the subject of quarantine restriction for decades (11). The pale potato cyst nematode $G$. pallida was first discovered in Idaho in 2006 (17). Since then, an extensive survey of the major potatogrowing acreage has been carried out to determine the extent of PCN distribution and to ensure that the appropriate steps are taken to prevent further infestation. Material suspected to contain PCN cysts has been analyzed by morphological and molecular means at the United States Department of Agriculture (USDA)-Agricultural Research Service Nematology Laboratory in Beltsville, MD.

Comprehensive descriptions of reference populations are key for accurate diagnosis of PCN and, ultimately, for preventing the spread of these nematodes. In real-world diagnostic scenarios, morphological and molecular approaches are essential, serving complementary diagnostic roles. Despite the increasing reliance

Corresponding author: A. M. Skantar;

E-mail address: andrea.skantar@ars.usda.gov

doi:10.1094/PHYTO-01-10-0010

This article is in the public domain and not copyrightable. It may be freely reprinted with customary crediting of the source. The American Phytopathological Society, 2011. upon molecular diagnostics, traditional morphological identification is an invaluable triage tool. Molecular assays for PCN that have been validated for use by state and federal diagnostic labs are primarily based upon amplification of the ribosomal internal transcribed spacer region (ITS rDNA). In addition to polymerase chain reaction restriction fragment length polymorphism (PCRRFLP) of the ITS region $(15,41,44)$ and multiplex PCR $(12,39)$, real-time PCR methods are emerging for rapid and sensitive detection of PCN $(23,36)$. However, these methods are subject to complications due to the presence of intraspecific or intrapopulation sequence variation. Bulman and Marshall (12) recognized the presence of ITS haplotypes in both G. pallida and $G$. rostochiensis and discussed the possible impact they might have on the effectiveness of species-specific multiplex PCR. Anthoine et al. (4) reported that molecular variation in Globodera spp. affected the reliability of several published molecular diagnostic tests. Although time consuming, sequence analysis is sometimes needed to provide additional information for PCN identification. Therefore, it is increasingly important to investigate new occurrences of Globodera spp. that do not fit the typical profiles, and to determine their potential impact on existing diagnostic procedures.

In 2008, cyst nematodes were found in soils collected from a Powell Butte, OR field with a cropping history including potato, wheat, other crops, and significant weed presence, and from two fields within Idaho. Herein, we describe the morphological and molecular characteristics of these populations. The significance of this research on the molecular methods used for diagnosis of PCN is discussed. 


\section{MATERIALS AND METHODS}

Morphological characterization. Juveniles for morphological observations were hatched from cysts that had been sieved from fresh soil and kept in water in Syracuse watch glasses in the laboratory. Juveniles were then fixed in 3\% formaldehyde and processed to glycerin by the formalin-glycerin method $(14,20)$. Cysts were similarly removed from soil samples by sieving, fixed for $12 \mathrm{~h}$ in $3 \%$ formaldehyde, and processed to glycerin. Photomicrographs of cyst vulval cones and fixed $\mathrm{J} 2$ were made with an automatic 35-mm camera attached to a Leica Wild MPS48 Leitz DMRB compound microscope, and measurements were made with an ocular micrometer on the same microscope. Some J2 heads and tail photomicrographs were taken on a Zeiss Ultraphot with DIC (differential interference contrast) and a Q-imaging Micropublisher 5 camera. Specimens were then morphologically identified with recent taxonomic keys and a compendium for identification of Globodera spp. $(5,13)$.

Molecular characterization. Molecular characterization of the populations included sequence-specific multiplex PCR $(12,39)$, PCR-RFLP of ITS1 and -2 rDNA $(7,37,38)$, and phylogenetic analysis of ITS1 and -2 rDNA sequences (41). Nematode juveniles or cysts were mechanically disrupted with a microscopy knife in $20 \mu \mathrm{l}$ of nematode extraction buffer (47) and stored at $-80^{\circ} \mathrm{C}$. Template preparation, PCR, restriction digests (RFLP), and purification of amplicon DNA were carried out as described previously (39). PCR products were cloned using the Strataclone PCR Cloning Kit (Stratagene, La Jolla, CA) according to the manufacturer's instructions. Plasmid clone DNA was prepared with the QiaPrep Spin Miniprep Kit and digested with EcoRI to verify the presence of the insert. Sequencing was performed at the University of Maryland Center for Biosystems Research. DNA from 14 ITS rDNA clones representing juveniles from three separate cysts from the Oregon soil sample were sequenced. Direct sequencing was performed on four ITS rDNA amplicons, representing four juveniles from the single available cyst of Idaho sample no. 167. DNA from 11 clones from Idaho sample no. 347, representing juveniles from two cysts, was sequenced. New sequences were submitted to the GenBank database at the National Center for Biotechnology Information (NCBI) under accession numbers GQ896542 to GQ896547.

DNA sequences were assembled using Sequencher 4.9 (Genecodes, Ann Arbor, MI). Alignments were made with Clustal W (48), with minor corrections made in GeneDoc (27). All phylogenetic procedures were implemented in PAUP* version $4.0 \mathrm{~b} 4 \mathrm{a}$ (42). G. achilleae was specified as the outgroup. JModelTest $(16,33,34)$ was used to generate parameters for constructing a distance tree. Using AIC criteria, the HKY85 model (18) was selected, involving a time-reversible process, nonuniform nucleotide distribution, and different transition and transversion rates $(\mathrm{HKY}+\mathrm{I}+\mathrm{G})$. The gamma shape parameter of 0.4440 for this model was implemented with the neighbor-joining (BioNJ) algorithm employing a minimum evolution objective function to produce a distance tree bootstrapped with 1,000 replications. A maximum parsimony tree was also constructed with 103 parsimony informative characters of 848 total characters, with fast, stepwise-addition and slow heuristic searches of 1,000 bootstrap replications with ACCTRAN (accelerated transormation) character-state optimization and TBR (tree bisection-reconnection) branch-swapping.

\section{RESULTS}

Morphological descriptions. Measurements of cysts and second-stage juveniles are shown in Table 1 and listed in micrometers unless stated otherwise.

Oregon population cysts. $(n=12)$. Cysts light brown to slightly dark brown in color, spherical to subspherical with a protruding neck. Anus prominent and visible in all specimens, often with a V-shaped mark (Fig. 1A and B). Subcrystalline layer absent. Vulval region intact or fenestrated with a single circumfenestrate opening occupying all part of vulval basin. Vulval bridge, underbridge, and bullae absent. However, occasionally bullae-like structures were noted in a few specimens (Fig. 1C and D). Cyst wall patterns were ridge-like with heavy punctuations (Fig. 1E and F).

Oregon population second-stage juveniles. $(n=57)$. Body vermiform, tapering at both extremities, more so posteriorly (Fig. 2A). Some specimens were folded four times within egg. Head rounded, slightly set off with prominent cephalic sclerotization, bearing four to five annules (Fig. 2B to E). Stylet short, well developed; basal knobs rounded with a greater height and with a forward projection on anterior side (Fig. 2C). Excretory pore located near the beginning of the basal esophageal bulb. Phasmids not observed. Tail tapering uniformly but abruptly narrows with a slight constriction near the posterior third of the hyaline portion, ending with a finely rounded to pointed terminus (Fig. $2 \mathrm{~F}$ to $\mathrm{J}$ ). Hyaline tail terminus with four to seven refractive bodies. Cuticular annulations distinct with more prominent annules near the tail terminus. Lateral field with occasional areolation, consisting of four incisures.

Oregon population males. Not detected.

Idaho population no. 167 cysts. $(n=2)$. Light brown color, spherical with a protruding neck (Fig. 3A). Cyst wall pattern ridge-like with heavy punctuations (Fig. 3B). Vulval region intact or fenestrated, with a single circumfenestrate opening that occupies all part of vulval basin (Fig. 3C and D). Whole fenestra length $=22.5$ to $25 \mu \mathrm{m}$; distance from nearest edge of fenestra to anus $=40$ to $52 \mu \mathrm{m}$; number of ridges between anus and nearest edge of fenestra $=10$ to 14 ; Granek's ratio $=1.8$ to 2.1 .

TABLE 1. Morphometrics of diagnostic characters of second-stage juveniles and cysts of Globodera sp. from Oregon ${ }^{\mathrm{a}}$

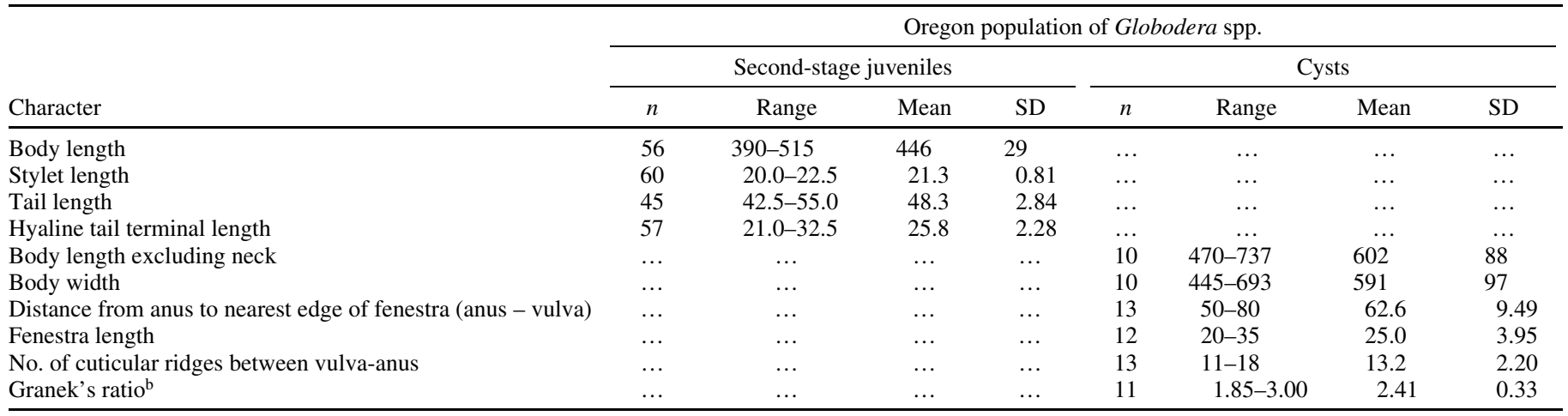

${ }^{\mathrm{a}} \mathrm{SD}=$ standard deviation.

b As modified by Hesling (19). 
Idaho population no. 167 second-stage juveniles. $(n=10)$. Body vermiform, specimens were folded four times within egg when cracked open. Tail tapering uniformly and usually bluntly rounded to pointed at the terminus (Figs. 3E to I and 4A). Tail abruptly narrowed, with a slight constriction near the posterior third of the hyaline portion, ending with a finely rounded to pointed terminus (Fig. 3J, M, and I). Lateral field with four incisures. Head rounded, slightly set off, with prominent cephalic sclerotization, bearing four annules. Stylet well developed; basal knobs rounded and with a forward projection on anterior side. Excretory pore located near the beginning of the basal esophageal bulb. Phasmids not observed. Length $=425$ to $485 \mu \mathrm{m}$ (451.7; SD 23.74); stylet $=21$ to $23 \mu \mathrm{m}(22.3$; SD 0.52$)$; tail $=42$ to $50 \mu \mathrm{m}$ (48; SD 3.09); hyaline tail terminus $=20$ to $30 \mu \mathrm{m}$ (24.6; SD 3.26).

Idaho population no. 347 cysts. $(n=2)$. Light brown color, spherical with a protruding neck. Cyst wall pattern ridge-like with heavy punctations. Vulval region intact or fenestrated, with a

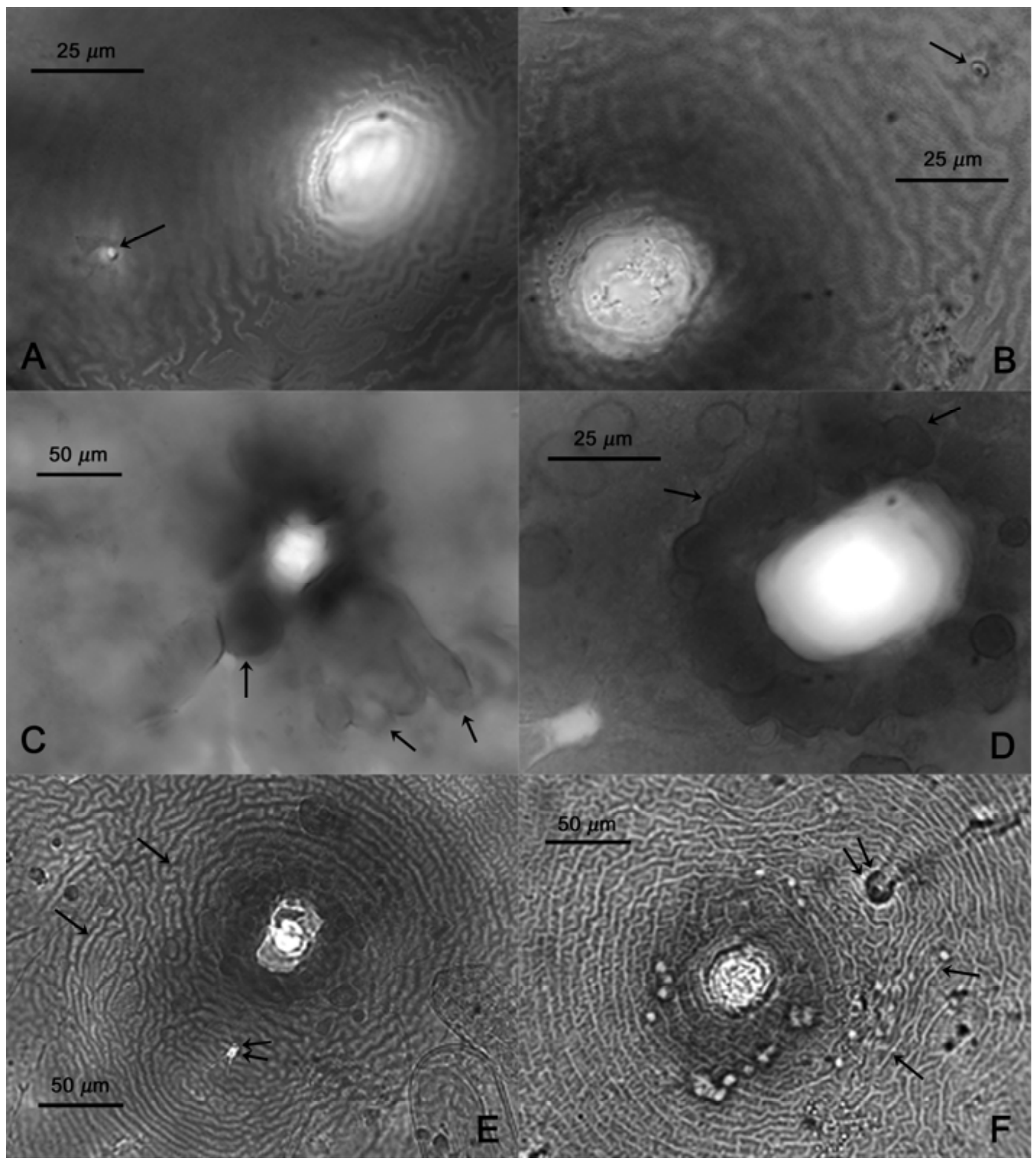

Fig. 1. Photomicrographs of terminal areas of cysts of the Globodera population from Oregon. Anal-vulval regions with arrows showing A and B, anal areas; $\mathbf{C}$ and $\mathbf{D}$, bullae-like structures; and $\mathbf{E}$ and $\mathbf{F}$, cyst wall patterns; and $\mathbf{E}$ and $\mathbf{F}$, double arrows showing anal areas. 
single circumfenestrate opening occupying all part of vulval basin. Whole fenestra length $=25 \mu \mathrm{m}$; distance from nearest edge of fenestra to anus $=30$ to $55 \mu \mathrm{m}$; number of ridges between anus and nearest edge of fenestra $=8$ to 14 ; Granek's ratio $=1.2$ to 2.2.
Idaho population no. 347 second-stage juveniles. $(n=8)$. Body vermiform, specimens were degraded and folded four times within egg when cracked open. Tail tapering uniformly and usually bluntly rounded to pointed at the terminus (Fig. 4B).

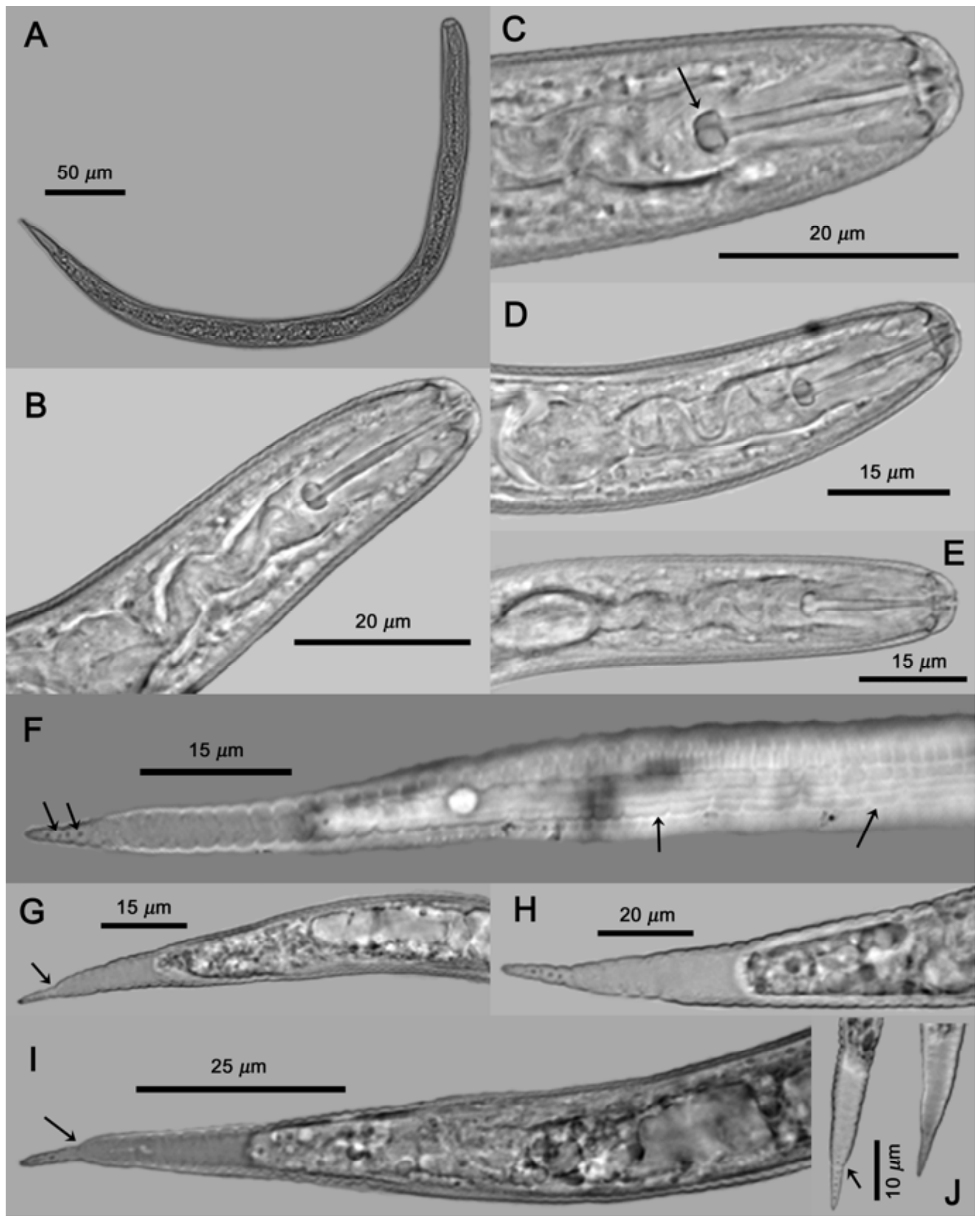

Fig. 2. Photomicrographs of second-stage juveniles of the Globodera population from Oregon. A, Whole juvenile; B to E, anterior regions showing stylet and part of esophagus; $\mathbf{C}$, high stylet knobs (arrow). F, Tail showing areolated nature of lateral field with four lines (right arrows) and refractive bodies in tail terminus (left arrows). $\mathbf{G}$ to $\mathbf{J}$, Tail variations; $\mathbf{G}, \mathbf{I}$, and $\mathbf{J}$, arrows showing abrupt narrowing of tail with slight constriction. 
Lateral field with four incisures. Head rounded, slightly set off with prominent cephalic sclerotization, bearing four annules. Stylet well developed; basal knobs rounded and with a forward projection on anterior side. Length 421 to $435 \mu \mathrm{m}$ (428; SD 9.89); stylet $=22$ to $23 \mu \mathrm{m}(22.5 ; \mathrm{SD} 0.32)$; tail $=48 \mu \mathrm{m}$; hyaline tail terminus $=22.5$ to $30 \mu \mathrm{m}(25.7 ;$ SD 2.52$)$.

Sample locality and repository. The Oregon population was collected in May 2008 during a mandated survey of a Powell

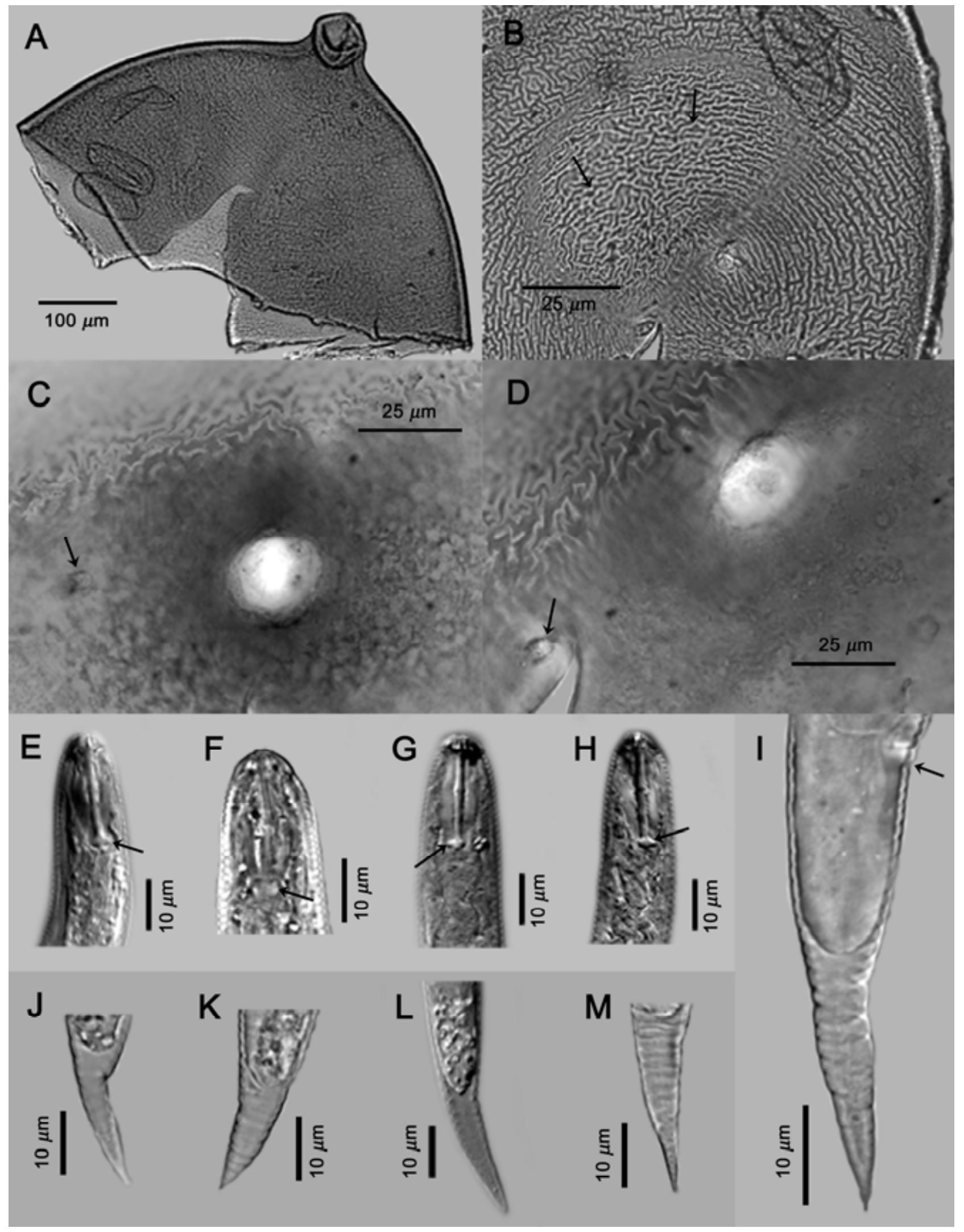

Fig. 3. Photomicrographs of cysts and second-stage juveniles of the Globodera population from Idaho. A, Anterior region. B to D, Anal-vulval regions: B, arrows showing cyst wall pattern; $\mathbf{C}$ and $\mathbf{D}$, arrows showing anal areas. $\mathbf{E}$ to $\mathbf{H}$, Anterior and posterior regions of second-stage juveniles; $\mathbf{E}$ to $\mathbf{G}$, arrows showing stylet knobs, with DIC images of E, G, and H. I to M, Tails with variable termini; DIC image of $\mathbf{L}$. Tail in I shows anal area (arrow). 
Butte, OR field with a history of potato, wheat, other crops, and weeds. Two additional populations, Idaho no. 167 and Idaho no. 347, were also detected during these surveys in August and October 2008. Idaho no. 167 came from a nonregulated field (location unknown) and Idaho no. 347 came from a field in Teton County.

Specimens were deposited in the USDA Nematode Collection, Beltsville, MD. For the Oregon population, second-stage juveniles, slide numbers were T-5847p to T-5862p and vial numbers T535p to T-541p; for cysts, slides numbers were T5863p to T-5870p. For Idaho populations, second-stage juveniles, slide numbers were T-5871p to T-5873p and vial numbers T-542p to T-543p; for cysts, slide numbers were T-5874p to T-5879p.
Morphological summary. These unusual populations are characterized as belonging to the genus Globodera and having second-stage juveniles with a body length of $446 \mu \mathrm{m}$ (390 to $515)$; stylet length $=21.3 \mu \mathrm{m}$ (20 to 22.5$)$, with rounded to high basal knobs with a forward projection on anterior side; areolated lateral field with four incisures; tail $=48 \mu \mathrm{m}$ (43 to 55) long, tapering uniformly but sometimes abruptly narrowing, with a pronounced to slight constriction near the posterior third of the hyaline portion, ending with a finely rounded to pointed terminus; hyaline tail terminus $=26 \mu \mathrm{m}(21$ to 33$)$; cysts spherical to subspherical; light to dark brown; circumfenestrate; number of cuticular ridges between vulva and anus = 13 (11 to 18$)$; and Granek's ratio $=2.4$ (1.9 to 3$)$.

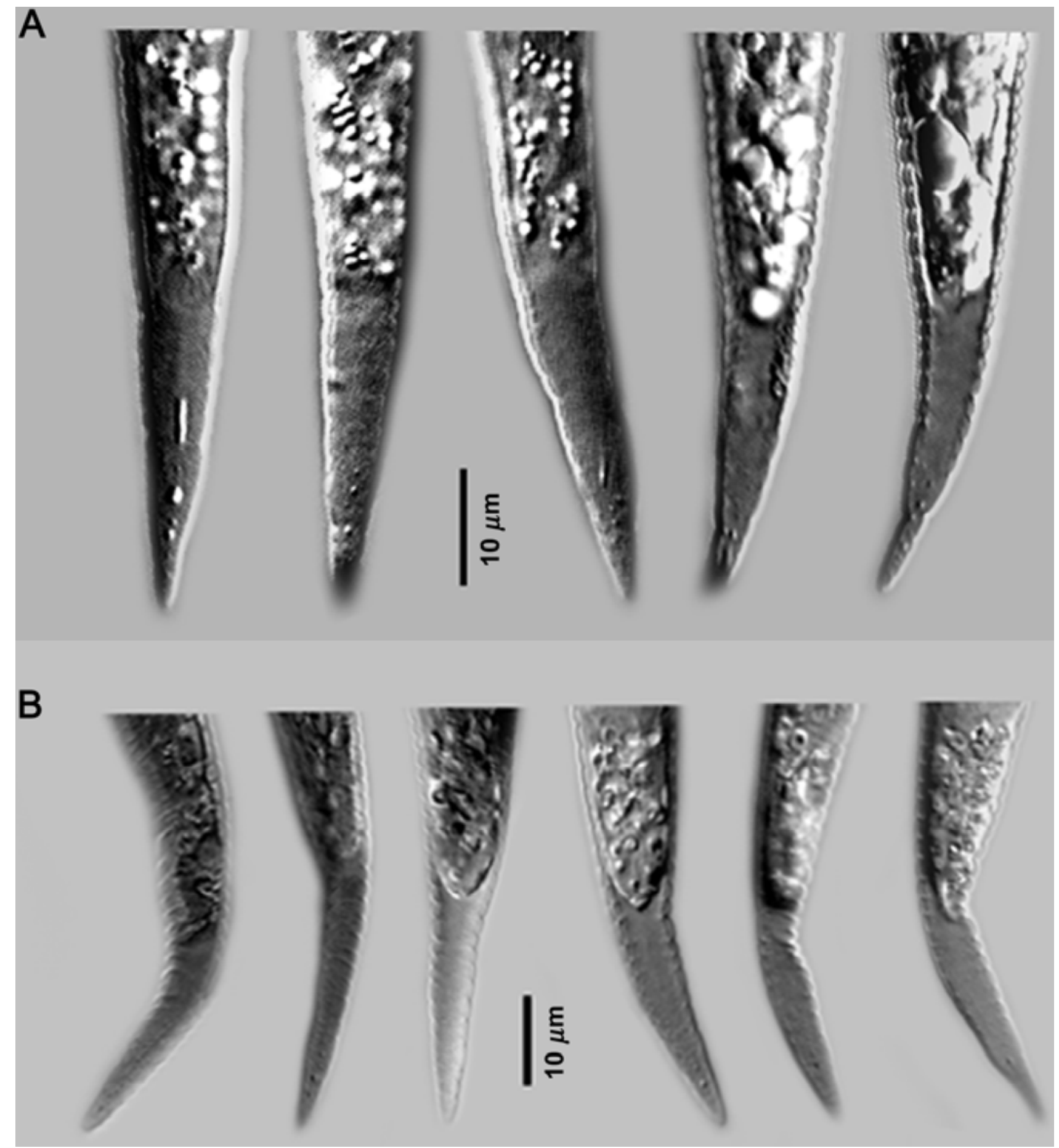

Fig. 4. Photomicrographs of DIC images of second-stage juvenile tails of the Globodera populations from A, Oregon and B, Idaho, showing morphological variation between unique distal constriction and bluntly pointed tails in both populations. 
ITS PCR and RFLP. Globodera spp. populations included in this study are listed in Table 2. DNA extracted from single juveniles of the Oregon population was subjected to conventional and multiplex PCR amplification of the ITS1 and -2 region. Amplifications with general ITS primers TW81 and AB28 resulted in PCR products of the expected size $(\approx 1,000 \mathrm{bp})$, consistent with those observed for G. pallida, G. rostochiensis, and G. tabacum (not shown). However, multiplex primers pITSp4, pITSr3, and pITS5 failed to amplify any product from the Oregon samples (Fig. 5A), while the control DNA from G. rostochiensis or G. pallida gave the expected products of 464 or $265 \mathrm{bp}$, respectively. When modified multiplex primer pITSt 4 was included with pITS5 and pITSr3 (37), amplification from the Oregon DNA resulted in a 265-bp amplicon that was consistent with G. tabacum and G. rostochiensis (Fig. 5B); the 434-bp band that is typical of $G$. rostochiensis was absent.

Discrimination of $G$. pallida and $G$. rostochiensis is typically accomplished by digestion of ITS PCR products with restriction enzymes AluI, RsaI, Sau3AI, or TaqI; the enzyme Bsh1236I is known to distinguish these species plus G. tabacum. When the ITS amplicons from the Oregon population of a Globodera sp. were subjected to Bsh1236I digestion, the banding pattern differed from those of G. pallida, G. rostochiensis, and G. tabacum, appearing instead as a mixed pattern with individual bands in common with each species (Fig. 5C). Digestion with RsaI also gave a distinct pattern for this population (data not shown). PCRRFLP of the two Idaho samples gave results similar to those of the Oregon specimens, in that the standard ITS PCR products were of the expected size $(\approx 1 \mathrm{~kb})$, the multiplex PCR results were similar to G. tabacum, and the RFLP patterns were mixed (data not shown).

DNA sequence analysis. Direct sequencing of purified ITS amplicons from the Oregon population resulted in numerous ambiguous base calls; therefore, 14 PCR product clones were selected for sequencing. Sequence divergence among these clones was as high as $2.8 \%$, with one clone (GQ896543) containing a unique 27-bp deletion. The few available J2 from Idaho sample no. 167 yielded PCR products with identical sequences that were obtained directly from the purified amplicons. For Idaho no. 347, 11 ITS rDNA clones were sequenced. Out of $977 \mathrm{bp}$, the clones exhibited a maximum divergence of $0.61 \%$. Alignment of sequences from all three populations revealed variation of 0 to $27 \mathrm{bp}$, with several identical clones and most deviating $<5 \mathrm{bp}$ from the consensus. Sequence divergence between $G$. pallida populations was previously reported to be $1.02 \%$, and divergence between clones within a population was $0.44 \%$ (41). Excluding the Oregon clone with the deletion, the degree of sequence divergence within these populations was similar to those reported for G. tabacum, G. rostochiensis, or G. pallida $(12,41)$.

Phylogenetic analysis. Compared with the NCBI database, sequences from the Oregon and Idaho populations were $98.7 \%$ identical to DQ097514, representing a G. pallida population from Argentina. DQ097514 differed from the individual clone sequences of the Oregon or Idaho populations at 5 to $15 \mathrm{bp}$. Representative ITS sequences from the Oregon or Idaho no. 347 clones and the consensus from Idaho no. 167 were aligned with several sequences from GenBank and unpublished data, with $G$. achilleae as the outgroup (Table 2). A neighbor-joining tree

TABLE 2. List of nematode species and populations used in this study

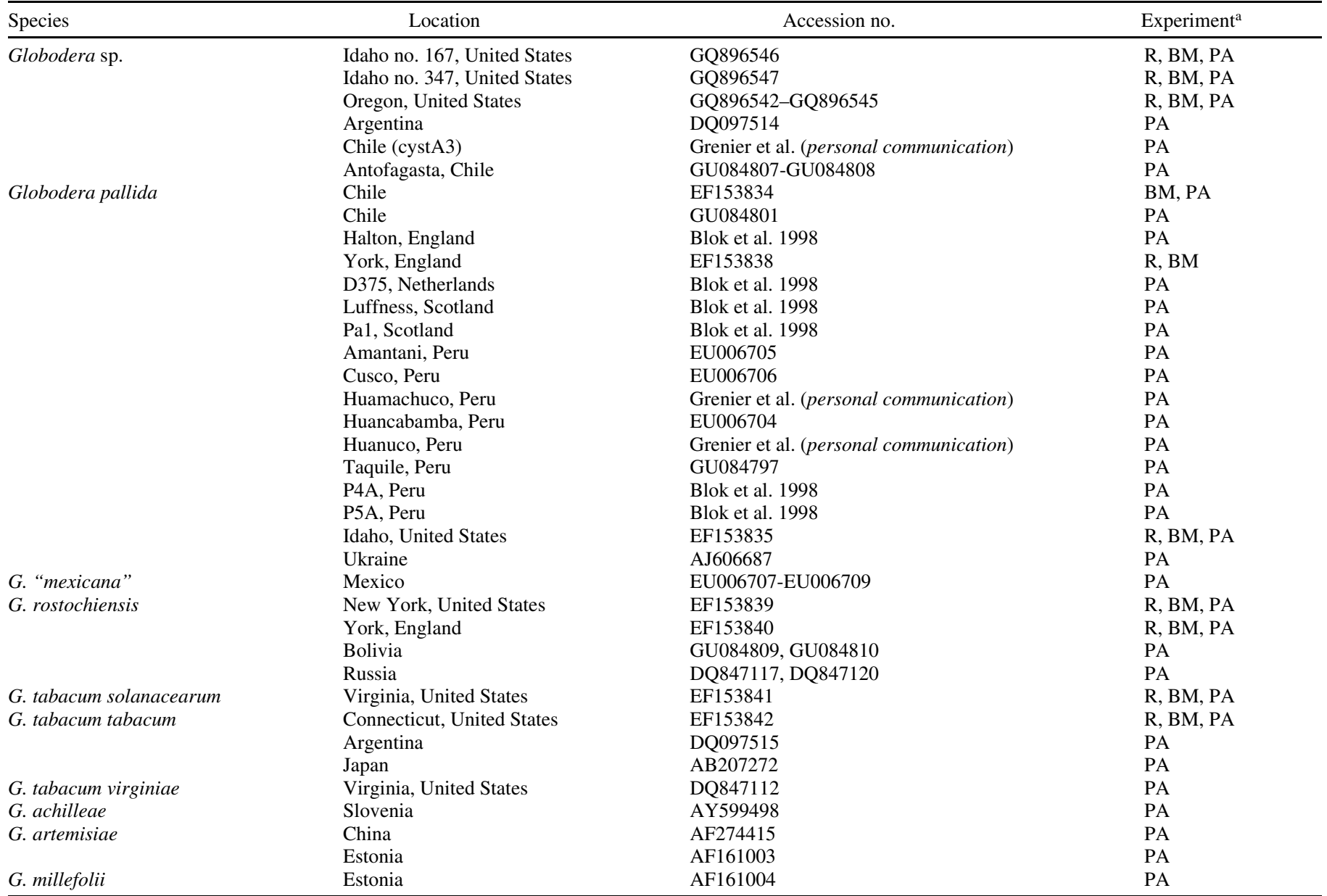

${ }^{\mathrm{a}} \mathrm{R}=$ restriction fragment length polymorphism study, $\mathrm{BM}=$ multiplex polymerase chain reaction assay using species-specific $\mathrm{rDNA}$ primers, and PA = phylogenetic and sequence analysis of the internal transcribed spacer (ITS) 1 and ITS2 rDNA region. 
showing relationships inferred from the ITS-rDNA gene is shown in Figure 6. The Oregon and Idaho populations fit into a highly supported clade $(93 \%)$ that included the Argentina sequence and unpublished sequences from a Chilean population (E. Grenier, personal communication). Within the Globodera sp. clade, two subclades of ITS haplotypes are apparent. The Globodera sp. clade formed a sister group to the clade containing $G$. rostochiensis and G. tabacum complex, although bootstrap support for
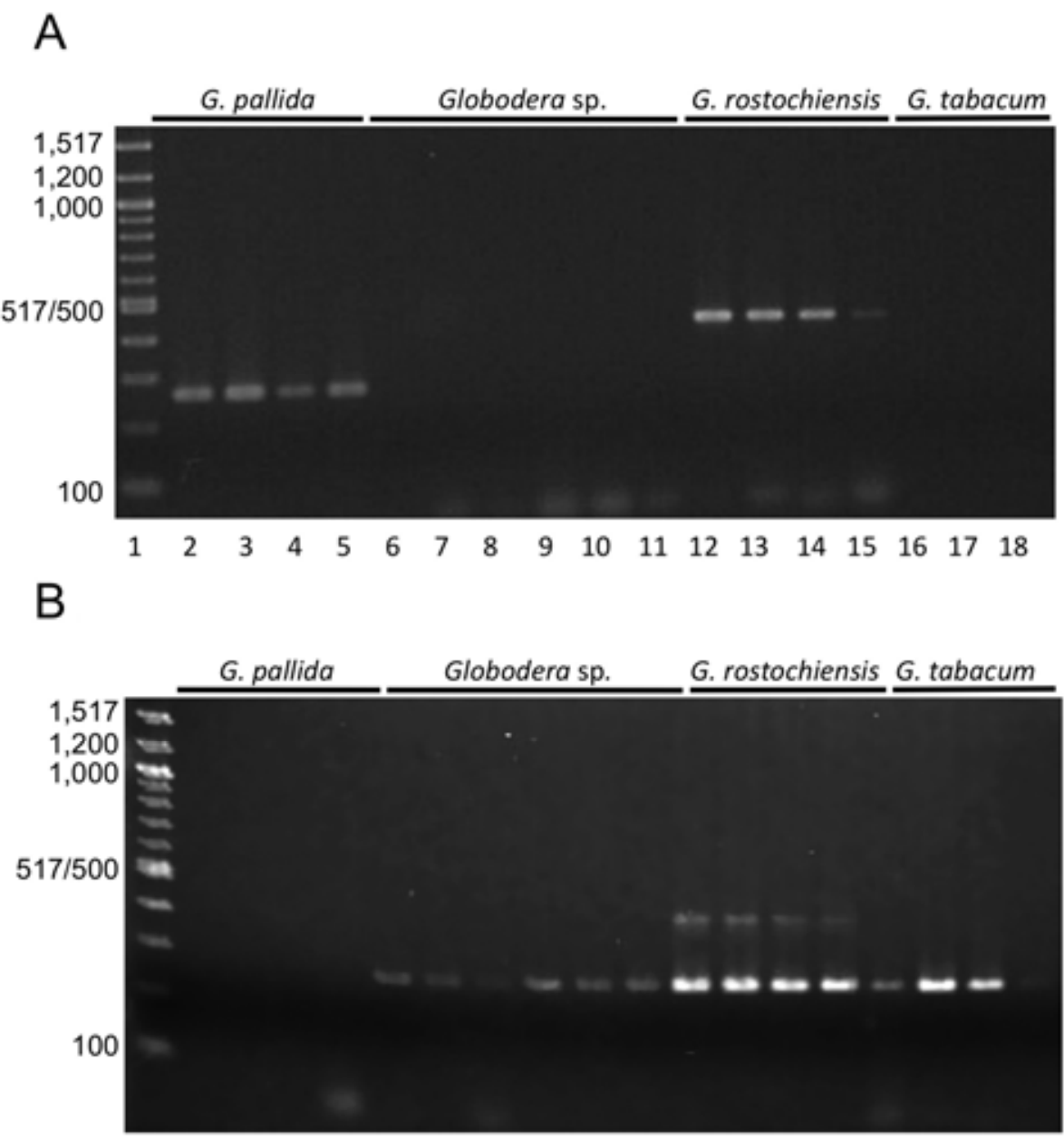

$\begin{array}{llllllllllllllllll}1 & 2 & 3 & 4 & 5 & 6 & 7 & 8 & 9 & 10 & 11 & 12 & 13 & 14 & 15 & 16 & 17 & 18\end{array}$

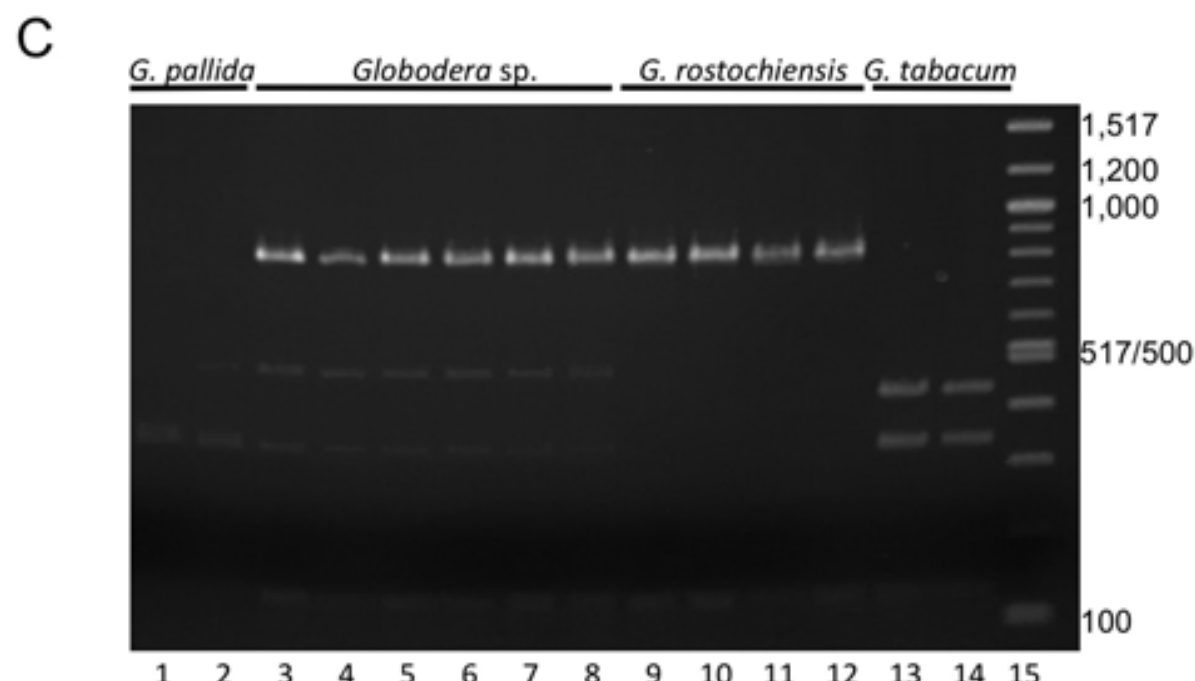

Fig. 5. Molecular analysis of the Globodera population. A, Multiplex polymerase chain reactions of internal transcribed spacer (ITS) rDNA from Globodera spp. using primers PITSr3, PITSp4, and ITS5. B, Multiplex polymerase chain reaction (PCR) of Globodera populations using primers PITSt4, PITSr3, and ITS5. A and B, Lane 1, 100-bp ladder (New England Biolabs, Ipswich, MA); lanes 2 and 3, Globodera pallida, Idaho; lanes 4 and 5, G. pallida, York, UK; lanes 6 to 11, G. sp., Oregon; lanes 12 and 13, G. rostochiensis, New York; lanes 14 and 15, G. rostochiensis, York, United Kingdom; lanes 16 and 17 , G. tabacum tabacum Connecticut; lane 18, G. tabacum solanacearum, Virginia. Numbers to the left indicate sizes of selected marker bands in the ladder. C, Amplified ITS PCR products from Globodera spp. digested by Bsh1236I. Lanes 1 and 2, G. pallida, York, United Kingdom; lanes 3 to 8, Globodera sp., Oregon; lanes 9 and 10, G. rostochiensis, NY; lanes 11 and 12, G. rostochiensis, United Kingdom; lanes 13 and 14, G. tabacum tabacum, CT; and lane 15, 100-bp ladder; sizes to the right. 
this position was modest (64\%). Bootstrap support was $99 \%$ for the G. tabacum clade and $80 \%$ for G. rostochiensis. Bootstrap support for the G. "mexicana" group was also high (100\%), although support for the overall grouping of G. pallida was weaker $(68 \%)$. The latter situation most likely reflects the molecular diver- sity of South American G. pallida populations that have been described in recent years. Maximum parsimony analysis (not shown) gave similar overall tree topologies, positioning the Globodera sp. clade as a sister group to the clade containing G. tabacum and $G$. rostochiensis, with relatively weak bootstrap support (60\%).

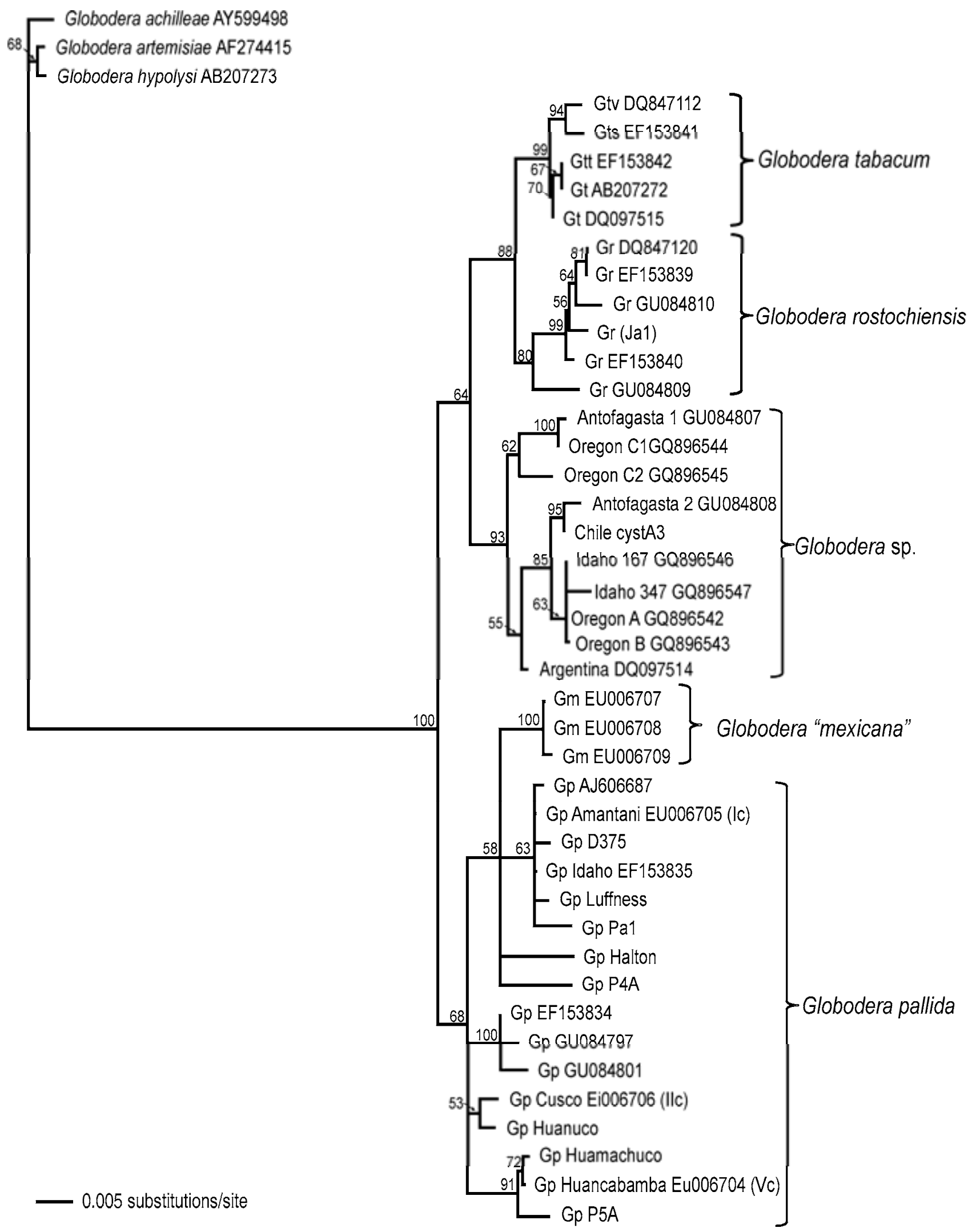

Fig. 6. Neighbor-joining phylogram of internal transcribed spacer (ITS) rDNA sequences from Oregon and Idaho populations and other Globodera spp. with outgroups Globodera achilleae, G. artemisiae, and G. hypolysi, and with bootstrap values from 1,000 replicates as implemented in PAUP* 4.0b10 (42). Bootstrap values $>50 \%$ are shown above branches. Roman numerals in parentheses indicate clade designations determined from cytochrome $b$ data (32). 


\section{DISCUSSION}

The described Globodera populations are morphologically variable, with some features outside the ranges expected for $G$. pallida, G. rostochiensis, or G. tabacum complex. Compared with G. pallida, the cyst body length is slightly longer, and the secondstage juvenile stylet length is slightly shorter. In some individuals, the stylet knob height is greater, the tail shape abruptly narrows, with a slight constriction near the posterior third of the hyaline tail terminus, and tail annules are more prominent. Compared with $G$. rostochiensis, the hyaline tail terminus has a large number of refractive bodies (four to seven). Cysts of the new populations have a smaller Granek's ratio and fewer cuticular ridges between the anus and vulva. In some individuals, the tail termini of second-stage juveniles are more bluntly pointed, and the stylet knobs are more anteriorly directed, with greater height. Unlike $G$. tabacum (Lownsbery \& Lownsbery 1954) Skarbilovich 1959, the cyst wall often lacks a network-like pattern and, in some individuals, the juvenile tail terminus distinctly narrows after a constriction. Approximately two-thirds of juvenile tails in the Oregon population and few from the Idaho population possessed the unique distal tail constriction and attenuation (Fig. 4).

Results of the multiplex PCR (Fig. 5A and B) and RFLP studies (Fig. 5C) can be explained in light of the DNA sequences obtained from the Oregon and Idaho populations. For instance, the 265-bp amplicon observed for the Globodera sp. populations (Fig. 5B) can be explained by the exact match between the ITS sequences for the Oregon and Idaho populations and the $G$. tabacum pITSt 4 primer. Also, in silico analysis of the ITS clone sequences revealed the existence of restriction site polymorphisms that are predicted to result in either three or four bands upon digestion with Bsh1236I. In comparison, European-type $G$. pallida sequences would be expected to give five fragments, $G$. rostochiensis, three; and G. tabacum, five (with fragments $<75$ not visible on a gel). As reflected in the different clone sequences recovered, loss of one or two Bsh1236I restriction sites should result in three or four fragments. The restriction patterns observed in Figure 5C were due to the presence of a mixture of ITS haplotypes within the single $\mathrm{J} 2$ used for each lane of the RFLP experiment. These results underscore the need for caution in interpreting the results of multiplex PCR or RFLP in a diagnostic setting, especially in circumstances where the population does not fit a "typical" profile. The development of a multiple marker, molecular diagnostic key for Globodera spp. may ultimately be necessary to compensate for molecular complexities encountered in any one marker (1).

Other than the DNA sequence in GenBank, there is little information available for the Argentina population. Cysts of this nematode were found on Solanum tuberosum subsp. andigena (also known as $S$. andigenum) in the Salta province of Northern Argentina, and the morphometrics and ITS rDNA reportedly fit best with $G$. pallida $(21,22)$. Unfortunately, no morphological comparisons with the Argentina population were possible, because there are no published photographs or morphometrics and this particular isolate was not maintained in culture. It should also be noted that the Chilean sequences (Antofagasta 1 and 2, and Chile cyst A3) are distinct from the Chilean G. pallida sequence EF153834 which, instead, groups with European populations and G. pallida from Idaho (EF153835). Further information about the Antofagasta population is expected in another study (E. Grenier, personal communication). However, it is interesting to note that Antofagasta, Chile is similar in latitude and within $500 \mathrm{~km}$ of Salta Province, Argentina, the presumptive source of the Globodera population represented by sequence DQ097514. Additional sampling of Globodera spp. from the entire Andean region would provide valuable clues to the relationship between populations from that diverse ecosystem and those from the United States.
The morphological and molecular plasticity observed in these populations has been previously described for the genus Globodera $(5,32,45,46)$. In one instance, a potato cyst nematode found in a soil sample from Scotland did not clearly fit with typical descriptions of either $G$. pallida or $G$. rostochiensis (38). Both long and short stylet lengths were found in juveniles from a single cyst. PCR-RFLP of the ITS region revealed unexpected restriction patterns, some of which appeared like G. pallida and some like $G$. rostochiensis. This unusual result was ultimately explained by sequence analysis, which revealed a 67-bp deletion in one clone and a restriction site-changing point mutation in another. Despite this variation, the population was not considered to represent a new species, because the phylogenetic analysis of ITS rDNA sequences placed them solidly in a clade with other European populations of $G$. pallida.

The existence of atypical PCNs has been demonstrated by a number of earlier molecular studies comparing $G$. pallida populations from South America with those from Europe. The uniquely virulent Peruvian isolate of pathotype P5A (29) was distinguished from European isolates by simple sequence repeat (SSR) analysis (8) and random amplified polymorphic DNA (RAPD) PCR $(6,9)$ and by digestion of ITS amplicons with MboI or TaqI (7). These results led to speculation that this population could represent a new species. In a phylogenetic study of worldwide Globodera populations inferred from ITS rDNA, Subbotin et al. (41) had found that P5A, along with Peruvian populations Huamachuco P6A, Huancabamba, and Cusco (GenBank accession numbers AF016866 to AF016868), formed a sister group to the major European clade, later found to also include the U.S. population from Idaho (39). Thus, Subbotin et al. (41) extended the earlier presumption about P5A by presenting that group as an unnamed new species. Taken in context with more recent information on these South American populations (32), P5A groups within the Vc subclade of G. pallida (Fig. 6). Therefore, the genetic diversity within the accepted species boundary of $G$. pallida appears to be expanding as more populations are characterized.

A comprehensive study, including RAPD analysis, ITS-RFLP, two-dimensional gel electrophoresis, and analysis of satellite DNA, confirmed the molecular separation of European G. pallida from several Peruvian populations, although the latter were not assigned unambiguously to any Globodera spp. (15). More recently, relationships of a larger assemblage of Peruvian isolates were established through phylogenetic analysis of microsatellite markers and mitochondrial cytochrome b (cyt b) sequences, revealing five major clades (30). Further analysis of microsatellite loci and cyt b data localized the geographic origin of European populations to a small area in southern Peru, and revealed a southto-north decrease in genetic diversity among these distinct clades $(31,32)$. Based on cyt b phylogeny, Pylypenko et al. (35) concluded that P5A was closely related to Peru populations Huancayo, Cajabama, and Otuzco (clade IVc) and most likely had a different geographic origin than the major group of European populations (clade Ic). Additional analysis of cyt $b$ and other markers from the Oregon population and nematodes from Argentina should shed more light on their genetic relationship to these Peruvian isolates.

The morphological and molecular variation observed in the Oregon and Idaho populations, together with the molecular separation of some South American Globodera populations, raises important philosophical and practical questions about precisely what constitutes a species and on the criteria used to define species boundaries (2). Globodera spp. have been historically classified on the basis of morphology, host specificity, and reproductive isolation, although some species assignments in the Heteroderidae that were based on these criteria have failed to remain valid, especially after evaluation by molecular methods. For example, G. solanacearum (Miller \& Gray 1972) Behrens 
1975 and G. virginiae (Miller \& Gray 1968) Stone 1973, once considered separate species, are now regarded as subspecies of $G$. tabacum, due largely to the genetic similarity demonstrated therein $(10,24,42,43)$. The morphological variation we observed within and among the Oregon and Idaho populations is unfortunately common for many species within the genus. The conclusion of Stone (40) that "No absolute criterion distinguishes even the most well-characterized species of the Globodera complex" thus remains true within the current morphological context.

Although some stress the importance of demonstrating reproductive isolation as a decisive factor for species delimitation, this presents a particular challenge for passively dispersing plantparasitic nematodes and sometimes conflicts with other criteria. Although viable hybrid populations have been created in the laboratory from crosses of G. rostochiensis, G. pallida, and G. tabacum (25), such hybridization may not happen in naturally occurring mixtures; for example, of $G$. pallida and $G$. rostochiensis (28). Moreover, mating experiments have not resolved the taxonomic status of G. "mexicana" Campos-Vela 1967. Viable and fertile hybrids were obtained from crosses of $G$. "mexicana" and $G$. pallida (26), consistent with their molecular similarity $(10,15)$ but despite their differences in host range $(45)$. Therefore, reluctance to separate some Peruvian clades of Globodera into distinct species is understandable in light of the viable offspring obtained from crosses between individuals from northern and southern Peruvian populations, and on the apparent lack of morphological diversity among them (30). However, the Globodera sp. clade appears genetically more distant from G. pallida than the Peruvian and European $G$. pallida clades are to each other, and more distant than G. pallida is from G. "mexicana" (Fig. 6).

Analyses of many Peruvian isolates have not included detailed morphological descriptions; therefore, the extent of anatomical variation they have compared with type specimens of G. pallida has not been demonstrated. Although the pathogenicity of some Peruvian populations on potato was previously described (15, 29,35), no clear correlation between molecular markers and virulence has been found. Perhaps morphological descriptions of the South American populations would help to further define the taxonomic criteria by which populations such as those from Oregon and Idaho should be evaluated.

Ultimately, the ability to reproduce on potato is the most important characteristic of Globodera populations with respect to the potential for economic impact. Preliminary greenhouse and laboratory studies comprising multiple observations indicate that the Oregon population reproduces on potato ( $S$. tuberosum). Further investigation of the morphology, molecular taxonomy, and pathogenicity of these Globodera populations is needed. Until alternative diagnostics markers that are unaffected by polymorphisms can be developed, ITS-based identification methods for Globodera spp. must be used with extreme caution. Sequence analysis of ITS rDNA or other markers may be necessary to resolve unclear or conflicting results obtained by existing ITSbased methods.

\section{ACKNOWLEDGMENTS}

We thank E. Grenier, INRA France, for sharing unpublished data; and M. Hult, D. Martel, L. Adams, and S. Ochs for excellent technical assistance. Mention of trade names or commercial products in this publication is solely for the purpose of providing specific information and does not imply recommendation or endorsement by the United States Department of Agriculture.

\section{LITERATURE CITED}

1. Adam, M. A. M., Phillips, M. S., and Blok, V. C. 2007. Molecular diagnostic key for identification of single juveniles of seven common and economically important species of root-knot nematode (Meloidogyne spp.). Plant Pathol. 56:190-197.

2. Adams, B. J. The species delimitation uncertainty principle. 2001. J. Nematol. 33:153-160.

3. Agricultural Statistics Board. 2009. Potatoes 2008 Summary Report. United States Department of Agriculture, National Agricultural Statistics Service.

4. Anthoine, G., Chappé, A. M., Fouville, D., Henriquez Flores, E., Mugniéry, D., and Grenier, E. 2008. Reliability and limits of published molecular tests for the specific identification of potato cyst nematodes (PCN) of quarantine concern. Page 125 in: (Abstr.) Proc. 5th Int. Congr. Nematol. Brisbane, Australia.

5. Baldwin, J. G., and Mundo-Ocampo, M. 1991. Heteroderinae, cyst- and non-cyst-forming nematodes. Pages 275-362 in: Manual of Agricultural Nematology. W. R. Nickle, ed. Marcel Dekker, New York.

6. Bendezu, I. F., Evans, K., Burrows, P. R., De Pomerai, D., and CantoSaenz, M. 1998. Inter and intra-specific genomic variability of the potato cyst nematodes Globodera pallida and G. rostochiensis from Europe and South America using RAPD-PCR. Nematologica 44:49-61.

7. Blok, V. C., Malloch, G., Harrower, B., Phillips, M. S., and Vrain, T. C. 1998. Intraspecific variation in ribosomal DNA in populations of the potato cyst nematode Globodera pallida. J. Nematol. 30:262-274.

8. Blok, V. C., and Philips, M. S. 1995. The use of repeat sequence primers for investigating genetic diversity between populations of potato cyst nematodes with differing virulence. Fundam. Appl. Nematol. 186:575582 .

9. Blok, V. C., Phillips, M. S., and Harrower, B. E. 1997. Comparison of British populations of potato cyst nematodes with populations from continental Europe and South America using RAPDs. Genome 40:286293.

10. Bossis, M., and Mugniéry, D. 1993. Specific status of six Globodera parasites of solanaceous plants studied by means of two-dimensional gel electrophoresis with a comparison of gel patterns by a computed system. Fundam. Appl. Nematol. 16:47-56.

11. Brodie, B. B., and Mai, W. F. 1989. Control of the golden nematode in the United States. Annu. Rev. Phytopathol. 27:443-461.

12. Bulman, S. R., and Marshall, J. W. 1997. Differentiation of Australasian potato cyst nematode (PCN) populations using the polymerase chain reaction (PCR). N. Z. J. Crop Hortic. Sci. 25:123-129.

13. Golden, A. M. 1986. Morphology and identification of cyst nematodes. Pages 23-45 in: Cyst Nematodes. F. Lamberti and C. E. Taylor, eds. Plenum Press, New York.

14. Golden, A. M. 1990. Preparation and mounting nematodes for microscopic observations. Pages 197-205 in: Plant Nematology Laboratory Manual. B. M. Zuckerman, W. F. Mai, and L. R. Krusberg, eds. University of Massachusetts Agricultural Experiment Station, Amherst.

15. Grenier, E., Bossis, M., Fouville, D., Renault, L., and Mugniéry, D. 2001. Molecular approaches to the taxonomic position of Peruvian potato cyst nematodes and gene pool similarities in indigenous and imported populations of Globodera. Heredity 86:277-290.

16. Guindon, S., and Gascuel, O. 2003. A simple, fast and accurate method to estimate large phylogenies by maximum likelihood. Syst. Biol. 52:696704.

17. Hafez, S. L., Sundararaj, P., Handoo, Z. A., Skantar, A. M., Carta, L. K., and Chitwood, D. J. 2007. First report of the pale cyst nematode, Globodera pallida, in the United States. Plant Dis. 91:325.

18. Hasegawa, M., Kishino, H., and Yano, T. 1985. Dating the human-ape split by a molecular clock of mitochondrial DNA. J. Mol. Evol. 22:160174.

19. Hesling, J. J. 1973. The estimation of Granek's ratio in round-cyst Heteroderas. Nematologica 19:119.

20. Hooper, D. J. 1970. Handling, fixing, staining and mounting nematodes. Pages 59-80 in: Laboratory Methods for Work with Plant and Soil Nematodes, 5th ed. J. F. Southey, ed. Her Majesty's Stationery Office, London.

21. Lax, P., Doucet, M. E., Gallardo, C., De L'Argentier, S. M., and Vilte, H. 2006. Plant-parasitic nematodes detected in Andean tubers from Argentina and Bolivia. Nematol. Bras. 30:195-201.

22. Lax, P., Manduric, S., Doucet, M. E., Gallardo, C., and De L'Argentier, S. M. 2005. Primera cita del nematodo blanco del quiste de la papa, Globodera pallida, en Argentina continental. (Abstr.) XIII LatinAmerican Congr. Plant Pathol. III Argentine Workshop of Plant Pathology. Carlos Pax, Cordoba, Argentina.

23. Madani, M., Subbotin, S. A., and Moens, M. 2005. Quantitative detection of the potato cyst nematode, Globodera pallida, and the beet cyst nematode, Heterodera schachtii, using real-time PCR with SYBR green I dye. Mol. Cell. Probes 19:81-86.

24. Marché, L., Valette, S., Grenier, E., and Mugniéry, D. 2001. Intra-species DNA polymorphism in the tobacco cyst-nematode complex (Globodera tabacum) using AFLP. Genome 44:941-946. 
25. Miller, L. I. 1983. Diversity of selected taxa of Globodera and Heterodera and their interspecific and intergeneric hybrids. Pages 207-220 in: Concepts in Nematode Systematics. The Systematics Association Special Volume No. 22. A. R. Stone, H. M Pratt, and L. F Khalil, eds. Academic Press, New York.

26. Mugniéry, D., Bossis, M., and Pierre, J. S. 1992. Hybridations entre Globodera rostochiensis Wollenweber G. pallida Stone, G. virginiae Miller \& Gray, G. solanacearum Miller \& Gray et " $G$. mexicana" Campos-Vela. Description et devenir des hybrides Fundam. Appl. Nematol. 15:375-382.

27. Nicholas, K. B., Nicholas, H. B., Jr., and Deerfield, D. W., II. 1997. GeneDoc: Analysis and visualization of genetic variation. EMBNEW.NEWS 4:1-4. http://www.nrbsc.org/gfx/genedoc/ebinet.htm.

28. Nijs, L. den. 1992. Interactions between Globodera rostochiensis and $G$. pallida in simultaneous infections on potatoes with different resistance properties. Fundam. Appl. Nematol. 15:173-178.

29. Phillips, M. S., and Trudgill, D. L. 1998. Variation of virulence, in terms of quantitative reproduction of Globodera pallida populations, from Europe and South America, in relation to resistance from Solanum vernei and S. tuberosum ssp. andigena CPC 2802. Nematologica 44:409-423.

30. Picard, D., Sempere, T., and Plantard, O. 2007. A northward colonisation of the Andes by the potato cyst nematode during geological times suggests multiple host-shifts from wild to cultivated potatoes. Mol. Phylogenet. Evol. 42:308-316.

31. Picard, D., Sempere, T., and Plantard, O. 2008. Direction and timing of uplift propagation in the Peruvian Andes deduced from molecular phylogenetics of highland biotaxa. Earth Planetary Sci. Lett. 271:326-336.

32. Plantard, O. Picard, D., Valette, S., Scurrah, M, Grenier, E., and Mugniéry, D. 2008. Origin and genetic diversity of Western European populations of the potato cyst nematode (Globodera pallida) inferred from mitochondrial sequences and microsatellite loci. Mol. Ecol. 17:2208-2218.

33. Posada, D. 2008. jModelTest: Phylogenetic model averaging. Mol. Biol. Evol. 25:1253-1256.

34. Posada, D., and Crandall, K. A. 2001. Selecting the best-fit model of nucleotide substitution. Syst. Biol. 50:580-601.

35. Pylypenko, L. A., Phillips, M. S., and Blok, V. C. 2008. Characterisation of two Ukrainian populations of Globodera pallida in terms of their virulence and mtDNA, and the biological assessment of a new resistant cultivar Vales Everest. Nematology 10:585-590.

36. Quader, M., Nambiar, L., and Cunnington, J. 2008. Conventional and real-time PCR-based species identification and diversity of potato cyst nematodes (Globodera spp.) from Victoria, Australia. Nematology 10:471-478

37. Radivojevic, M., Krnjaic, D., Krnjaic, S., Bacic, J., Subbotin, S. A., Madani, M., and Moens, M. 2001. Molecular methods confirming the presence of Globodera rostochiensis (Wollenweber, 1923) in Yugoslavia. Russ. J. Nematol. 9:139-141.

38. Reid, A., and Pickup, J. 2005. Molecular characterization of a morphologically unusual potato cyst nematode. Bull. OEPP/EPPO Bull. 35:69-72.

39. Skantar, A. M., Handoo, Z. A., Carta, L. K., and Chitwood, D. J. 2007. Morphological and molecular identification of Globodera pallida associated with potato in Idaho. J. Nematol. 39:133-144.

40. Stone, A. R. 1983. Three approaches to the status of a species complex, with a revision of some species of Globodera (Nematoda: Heteroderidae). Pages 221-233 in: Concepts in Nematode Systematics. The Systematics Association Special Volume No. 22. A. R. Stone, H. M Pratt, and L. F Khalil, eds. Academic Press, New York.

41. Subbotin, S. A., Halford, P. D., Warry, A., and Perry, R. N. 2000. Variations in ribosomal DNA sequences and phylogeny of Globodera parasitizing solanaceous plants. Nematology 2:591-604.

42. Swofford, D. L. 1998. PAUP* Phylogenetic Analysis Using Parsimony (*and other methods). Version 4.0. Sinauer Associates, Sunderland, MA

43. Syracuse, A. J., Johnson, C. S., Eisenback, J. D., Nessler, C. L., and Smith, E. P. 2004. Intraspecific variability within Globodera tabacum solanacearum using random amplified polymorphic DNA. J. Nematol. 36:433-439.

44. Szalanski, A. L., Sui, D. D., Harris, T. S., and Powers, T. O. 1997. Identification of cyst nematodes of agronomic and regulatory concern by PCR-RFLP of ITS1. J. Nematol. 29:255-267.

45. Thiéry, M., Fouville, D., and Mugniéry, D. 1997. Intra- and interspecific variability in Globodera, parasites of Solanaceous plants, revealed by Random Amplified Polymorphic DNA (RAPD) and correlation with biological features. Fundam. Appl. Nematol. 20:495-504.

46. Thiéry, M., and Mugniéry, D. 1996. Interspecific rDNA restriction fragment length polymorphism in Globodera species, parasites of Solanaceous plants. Fundam. Appl. Nematol. 19:471-479.

47. Thomas, W. K., Vida, J. T., Frisse, L. M., Mundo, M., and Baldwin, J. G. 1997. DNA sequences from formalin-fixed nematodes: Integrating molecular and morphological approaches to taxonomy. J. Nematol. 29:250-254.

48. Thompson, J. D., Higgins, D. G., and Gibson, T. J. 1994. CLUSTAL W: Improving the sensitivity of progressive multiple sequence alignment through sequence weighting, position-specific gap penalties and weight matrix choice. Nucleic Acids Res. 22:4673-4680. 